Pacific Journal of Mathematics

ABSTRACTIX SPLIT GRo OP EXTENSIO 


\title{
ABSTRACTLY SPLIT GROUP EXTENSIONS
}

\author{
G. HochsChild AND D. Wigner
}

1. Introductory survey. Consider a group extension $1 \rightarrow A \rightarrow E \underset{\pi}{\rightarrow} G \rightarrow 1$ in some category of groups with superstructure (topological, analytic, algebraic). Suppose it is split in the category of abstract groups, i.e., there is a homomorphism $\sigma: G \rightarrow E$ of abstract groups such that $\pi \circ \sigma$ is the identity map on $G$. We are concerned with the question of when it is possible to conclude that the extension is split as an extension in the given category.

The most surprising known result in this connection is due to $\mathrm{C}$. Moore [2, Th. 2.3]. It says that if the given category is that of locally compact separable topological groups, if $A$ lies in the center of $G$ and $G$ coincides with its commutator group $[G, G]$, then every $\sigma$ as above is necessarily continuous.

A more transparent situation in which our question has a positive answer is the following. Suppose the given extension is in the category of locally compact separable topological groups, that $A$ is a finitedimensional real vector group and that $G$ has a discrete subgroup $K$ such that the coset space $G / K$ is compact. Then, if an abstract split $\sigma$ exists, it follows that there is also a continuous split. In fact, our assumptions on $A$ and the topology of $G$ imply that the given extension has a continuous cross-section [3, Th. 12.2], and [0, Th. 4.2]. This yields a continuous 2-cocycle $f$ for $G$ in $A$ such that the extension is split if and only if $f$ is the coboundary of a continuous map from $G$ to $A$. By a well-known result due to van Est $[4, \S 4]$, the restriction map from the continuous cohomology of $G$ in $A$ to that of $K$ in $A$ is injective. Our assumption that the extension has an abstract split evidently implies that the cohomology class of $f$ is in the kernel of the restriction map. Therefore, it must be the 0-class, so that our extension has a continuous split.

Another positive case is that of an extension in the category of connected (real or complex) Lie groups in which the image $G$ is simply connected and the kernel $A$ is a central vector group. The existence of an abstract split $\sigma$ evidently implies that $A \cap[E, E]=1$, so that the given extension yields the extension

$$
1 \rightarrow A \rightarrow E /[E, E] \rightarrow G /[G, G] \rightarrow 1
$$

in the natural way. Since $G$ is simply connected, $[G, G]$ is closed in $G$, and $G /[G, G]$ is simply connected [1, Ch. XII, Th. 1.2]. As before, we 
have from [3, Th. 12.2] that the given extension has a continuous cross-section, whence we find that $E$ is simply connected. As just now, it follows that $[E, E]$ is closed in $E$, and $E /[E, E]$ is simply connected. Thus, the above extension is simply an extension of vector groups and therefore has a continuous (linear) split $\tau: G /[G, G] \rightarrow E /[E, E]$. Let $P$ denote the inverse image of $\tau(G /[G, G])$ in $E$, so that $P$ is a closed subgroup of $E$ containing $[E, E]$, and $P /[E, E]=\tau(G /[G, G])$. Now it is easy to check that $E$ is the direct product $A \times P$, which shows that the given group extension is split in the category of connected Lie groups.

The simplest example of an abstractly split extension of topological groups that is not continuously split, which must be known to many, is the following. Let $\mathscr{R}, \mathscr{Q}, \mathscr{Z}$ denote the additive groups of real numbers, rational numbers, integers, respectively. Let $\pi: \mathscr{R} \times(\mathscr{Q} / \mathscr{Z}) \rightarrow \mathscr{R} / \mathscr{Z}$ be defined by $\pi(x, y)=(x+\mathscr{Z})+y$. Clearly, $\pi$ is a continuous open homomorphism. The only compact subgroups of $\mathscr{R} \times(\mathscr{Q} / \mathscr{Z})$ are the finite subgroups of $\mathscr{Q} / \mathscr{Z}$, whence it is clear that the group extension given by $\pi$ has no continuous split. However, from a 2 -space decomposition $\mathscr{R}=S \oplus \mathscr{Q}$, we obtain the group decomposition $\mathscr{R} / \mathscr{Z}=S \times(\mathscr{Q} / \mathscr{Z})$, and hence an abstract group split $\sigma: \mathscr{R} / \mathscr{Z} \rightarrow \mathscr{R} \times(\mathscr{Q} / \mathscr{Z})$.

This example is not satisfactory, because of the lack of connectedness. In the categories of connected Lie groups and connected affine algebraic groups, our question leads to interesting subquestions by various further specialisations. In the positive direction, we shall make some progress for unipotent affine algebraic groups over fields of characteristic 0 . In the negative direction, we shall see how abstractly, but not continuously, split extensions of connected Lie groups arise from the fundamental group of the image group. The question of the existence of such examples, with simply connected image group, remains unresolved.

With regard to the above and to what follows, it is a pleasure to acknowledge the benefits we had from exploratory discussions with Brian Peterson and Chih-Han Sah.

2. Deflated extensions. A source of examples of the kind alluded to just above is the following construction. Let $H$ be a topological group, and let $K$ be a discrete central subgroup of $H$. Let $\tau$ be a homomorphism from $K$ to an abelian topological group $A$. Let $C$ be the subgroup of the direct product $A \times H$ consisting of the elements $(\tau(k), k)$, with $k$ in $K$. Clearly, $C$ is a discrete central subgroup of $A \times H$. Write $E$ for $(A \times H) / C$, and let $\eta: H \rightarrow H / K$ be the canonical homomorphism. The composite with $\eta$ of the projection $A \times H \rightarrow H$ induces a continuous, open and surjective homomorphism $\pi: E \rightarrow H / K$ whose kernel may be identified with $A$ in the evident way. It is easy to 
verify that the group extension determined by $\pi$ has an abstract split $H / K \rightarrow E$ if and only if $\tau$ is the restriction to $K$ of an abstract group homomorphism $H \rightarrow A$, and that it has a continuous split if and only if $\tau$ is the restriction of a continuous group homomorphism $H \rightarrow A$.

Here is the simplest specific example arising in this way. Let $M$ be the group of real matrices

$$
[\alpha, \beta, \gamma]=\left(\begin{array}{lll}
1 & 0 & 0 \\
\alpha & 1 & 0 \\
\gamma & \beta & 1
\end{array}\right)
$$

Put $H=\mathscr{R} \times M$. Now fix an irrational real number $\mu$, and let $K$ be the discrete central subgroup of $H$ consisting of the elements $(a+\mu b,[0,0, b])$, where $a$ and $b$ range over $\mathscr{Z}$. Finally, let $A=\mathscr{R}$, and define the homomorphism $\tau: K \rightarrow \mathscr{R}$ by

$$
\tau(a+\mu b,[0,0, b])=a+b
$$

We claim that the resulting extension of topological groups

$$
0 \rightarrow \mathscr{R} \rightarrow E \underset{\pi}{\rightarrow} H / K \rightarrow 1
$$

has an abstract split, but does not have a continuous split. It is evident that $\tau$ can be extended to an abstract group homomorphism $H \rightarrow \mathscr{R}$ (annihilating $M$ ). Therefore, our group extension has an abstract split.

Now suppose that, contrary to our claim, there is a continuous split of our group extension. As stated above, this yields a continuous homomorphism $f: H \rightarrow \mathscr{R}$ whose restriction to $K$ coincides with $\tau$. We have $K \subset \mathscr{R} \times[M, M]$. Now note that $f$ annihilates $[M, M]$ and is linear on the factor $\mathscr{R}$. It follows that there is a real number $\rho$ such that

$$
a+b=\tau(a+\mu b,[0,0, b])=\rho a+\rho \mu b .
$$

This gives the contradiction $\mu=1$, so that our claim is established.

3. Unipotent groups. We consider the category of unipotent affine algebraic $F$-groups, where $F$ is a field of characteristic 0 . Our results will automatically hold also in the category of simply connected nilpotent real or complex analytic groups. We denote the Lie algebra of a group $G$ by $\mathscr{L}(G)$. Recall that there are mutually inverse polynomial maps $\exp _{G}: \mathscr{L}(G) \rightarrow G$ and $\log _{G}: G \rightarrow \mathscr{L}(G)$, through which the categories of unipotent affine algebraic $F$-groups and of finitedimensional nilpotent $F$-Lie algebras are equivalent. Our question can be transferred to the category of nilpotent Lie algebras by virtue of the following proposition. 
Proposition. Let $G$ and $H$ be unipotent affine algebraic $F$-groups, with $F$ of characteristic 0 . Let $\gamma: G \rightarrow H$ be an abstract group homomorphism, and define the map $\gamma^{\prime}: \mathscr{L}(G) \rightarrow \mathscr{L}(H)$ by $\gamma^{\prime}=$ $\log _{H} \circ \gamma \circ \exp _{G}$. Then $\gamma^{\prime}$ is a morphism of 2 -Lie algebras. In this way, the abstract group homomorphisms $G \rightarrow H$ are in bijective correspondence with the morphisms of 2 -Lie algebras $\mathscr{L}(G) \rightarrow \mathscr{L}(H)$.

Proof. If $u$ and $v$ are Lie algebra elements such that $[u, v]=0$ then $\exp (u) \exp (v)=\exp (u+v)$. Using this with $\exp _{G}$ and $\exp _{H}$, we find that if $n$ is an integer and $x$ an element of $\mathscr{L}(G)$ then $\exp _{H}\left(\gamma^{\prime}(n x)\right)=$ $\exp _{H}\left(n \gamma^{\prime}(x)\right)$. Hence $\gamma^{\prime}(n x)=n \gamma^{\prime}(x)$. It follows that $\gamma^{\prime}(q x)=q \gamma^{\prime}(x)$ for every rational number $q$.

Since $\mathscr{L}(G)$ and $\mathscr{L}(H)$ are nilpotent, we can express products of exponentials in $G$ and $H$ by means of the Campbell-Hausdorff formula. This formula provides a set of rational numbers, indexed by finite sequences of 0 's and 1 's, such that the following holds. If $u$ and $v$ are given elements of $\mathscr{L}(G)$ or $\mathscr{L}(H)$, one attaches to each finite sequence of 0 's and 1's a certain multiple commutator of $u$ and $v$, according to the following recipe. To the sequence 0 we attach $u$, to the sequence 1 we attach $v$. Generally, if $[s]$ denotes the commutator attached to the sequence $s$, then $[0 s]=[u,[s]]$ and $[1 s]=[v,[s]]$. Since our Lie algebras are nilpotent, we have $[s]=0$ whenever the length of $s$ exceeds a certain bound. Therefore, if $q(s)$ is the rational number corresponding to $s$ in the Campbell-Hausdorff formula, the sum $\Sigma_{s} q(s)[s]$ is defined as an element of the Lie algebra. Denoting this by $\eta(u, v)$, we have $\exp (u) \exp (v)=\exp (\eta(u, v))$. We recall that if $\eta_{n}$ is the part of $\eta$ coming from the sequences of length $n$, then $\eta_{1}(u, v)=$ $u+v$ and $\eta_{2}(u, v)=\frac{1}{2}[u, v]$.

There is a sequence

$$
(0)=Z_{0} \subset \cdots \subset Z_{n}=\mathscr{L}(G)
$$

of ideals of $\mathscr{L}(G)$ such that $\left[\mathscr{L}(G), Z_{k+1}\right] \subset Z_{k}$ for $k=0, \cdots, n-1$. Now suppose we have already shown that $\gamma^{\prime}(u+v)=\gamma^{\prime}(u)+\gamma^{\prime}(v)$ and $\gamma^{\prime}([u, v])=\left[\gamma^{\prime}(u), \gamma^{\prime}(v)\right]$ whenever $u$ is in $\mathscr{L}(G)$ and $v$ is in $Z_{k}$. Let $q$ be a rational number, $v$ an element of $Z_{k+1}$ and $u$ any element of $\mathscr{L}(G)$. From the definitions, we have

$$
\gamma^{\prime}(\eta(q u, q v))=\eta\left(\gamma^{\prime}(q u), \gamma^{\prime}(q v)\right)
$$

This may be written

$$
\gamma^{\prime}\left(\Sigma_{k} q^{k} \eta_{k}(u, v)\right)=\Sigma_{k} q^{k} \eta_{k}\left(\gamma^{\prime}(u), \gamma^{\prime}(v)\right)
$$


Since, for $k>1, \eta_{k}(u, v)$ lies in $Z_{k}$ we may apply our inductive hypothesis to expand the left side, and we obtain

$$
\Sigma_{k} q^{k} \gamma^{\prime}\left(\eta_{k}(u, v)\right)=\Sigma_{k} q^{k} \eta_{k}\left(\gamma^{\prime}(u), \gamma^{\prime}(v)\right)
$$

Since this holds for all rational numbers $q$, the coefficients of $q^{k}$ on the two sides must be equal. In particular, taking $k=1$ and $k=2$, we obtain $\gamma^{\prime}(u+v)=\gamma^{\prime}(u)+\gamma^{\prime}(v)$ and $\gamma^{\prime}([u, v])=\left[\gamma^{\prime}(u), \gamma^{\prime}(v)\right]$. This proves, inductively, that $\gamma^{\prime}$ is a morphism of $\mathscr{Q}$-Lie algebras.

Conversely, suppose that $\delta: \mathscr{L}(G) \rightarrow \mathscr{L}(H)$ is a morphism of 2 -Lie algebras. Put $\gamma=\exp _{H} \circ \delta \circ \log _{G}$. Applying the Campbell-Hausdorff formula and noting that $\eta$ has rational coefficients, one verifies directly that $\gamma: G \rightarrow H$ is a homomorphism of abstract groups. Clearly, $\gamma^{\prime}=$ $\delta$. This completes the proof of the proposition.

If $L$ is an $F$-Lie algebra, $K$ a finite algebraic extension field of 2 contained in $F$ and $M$ a $K$-Lie algebra such that $L=M \otimes_{K} F$, then we call $M$ an absolutely algebraic form of $L$.

THEOREM. Let $F$ be a field of characteristic 0 , and let $G$ be a unipotent affine algebraic $F$-group. Suppose that $\mathscr{L}(G)$ has an absolutely algebraic form. Let $1 \rightarrow A \rightarrow E \rightarrow G \rightarrow 1$ be a group extension of unipotent affine algebraic $F$-groups having an abstract split. Then this group extension is split in the category of affine algebraic F-groups.

Proof. Write $\mathscr{L}(G)=L=M \bigotimes_{K} F$, as above. Viewing $M$ as a 2-Lie algebra, consider the extension of $K$-Lie algebras

$$
0 \rightarrow P \rightarrow M \otimes_{2} K \underset{\pi}{\rightarrow} M \rightarrow 0
$$

coming from the $K$-space structure of $M$. Write $U$ for $M \otimes_{2} K$, and note that $U$ is a two-sided $K$-module, with

$$
c \cdot(m \otimes k)=(\mathrm{cm}) \otimes k \quad \text { and } \quad(m \otimes k) \cdot c=m \otimes(k c)
$$

for $c$ and $k$ in $K$ and $m$ in $M$. The kernel $P$ of $\pi$ is clearly the two-sided $K$-submodule consisting of all sums of elements of the form $c \cdot u-u \cdot c$, with $u$ in $U$ and $c$ in $K$. Now $K$ is a finite-dimensional separable 2 -algebra, so that $K \otimes_{2} K$ is a finite-dimensional semisimple 2 -algebra, whence every two-sided $K$-module is semisimple. Let $S$ be a two-sided $K$-module complement of $P$ in $U$. Clearly, $c \cdot s=s \cdot c$ for every $s$ in $S$ and every $c$ in $K$. Let $T$ denote the two-sided $K$-submodule of $U$ consisting of all elements $u$ for which $c \cdot u=u \cdot c$ for all $c$ in $K$. We 
claim that $S=T$. In order to prove this, it suffices to show that $P \cap T=(0)$. By the semisimplicity, $P$ is a direct two-sided $K$-module sum $(P \cap T) \oplus R$. Since $U=P+T$, it follows from the definition of $P$ that every element of $P$ is a sum of elements $c \cdot p-p \cdot c$ with $c$ in $K$ and $p$ in $P$ (not only in $U$ ), and the above decomposition of $P$ shows that we may even take the elements $p$ to be in $R$. But this gives $P=R$, so that $P \cap T=(0)$.

Thus, $U=P \oplus T$. Evidently, $T$ is an ideal of $U$, so that this is a direct $K$-Lie algebra decomposition. The restriction of $\pi$ to $T$ is an isomorphism of $K$-Lie algebras $T \rightarrow M$. Let $\mu: M \rightarrow T$ be its inverse. By tensoring with $F$ and evident identifications, $\mu$ yields a morphism of $F$-Lie algebras

$$
\mu^{*}: L=M \bigotimes_{K} F \rightarrow T \bigotimes_{K} F \subset U \bigotimes_{K} F=M \bigotimes_{2} F \subset L \bigotimes_{2} F
$$

If $\tau: L \bigotimes_{2} F \rightarrow L$ is the morphism of $F$-Lie algebras coming from the $F$-space structure of $L$, it follows from the definition of $\mu^{*}$ that $\tau \circ \mu^{*}$ is the identity map on $L$.

Now let $1 \rightarrow A \rightarrow E \underset{\rho}{\longrightarrow} G \rightarrow 1$ be as in the statement of the theorem. This yields the extension of $F$-Lie algebras

$$
0 \rightarrow \mathscr{L}(A) \rightarrow \mathscr{L}(E) \underset{\rho^{\prime}}{\longrightarrow} \mathscr{L}(G) \rightarrow 0
$$

By our above proposition, an abstract split of the given group extension yields a morphism of 2 -Lie algebras

$$
\sigma: \mathscr{L}(G) \rightarrow \mathscr{L}(E)
$$

such that $\rho^{\prime} \circ \sigma$ is the identity map on $\mathscr{L}(G)$. By tensoring with $F$, we obtain the morphism of $F$-Lie algebras

$$
\sigma^{*}: \mathscr{L}(G) \bigotimes_{2} F \rightarrow \mathscr{L}(E) \bigotimes_{2} F
$$

Let $\gamma: \mathscr{L}(E) \otimes_{2} F \rightarrow \mathscr{L}(E)$ be the morphism of $F$-Lie algebras coming from the $F$-space structure of $\mathscr{L}(E)$. Then, if $\mu^{*}$ is the morphism of $F$-Lie algebras obtained above, the composite

$$
\gamma \circ \sigma^{*} \circ \mu^{*}: \mathscr{L}(G) \rightarrow \mathscr{L}(E)
$$

is a split of our above extension of $F$-Lie algebras. Via $\log _{G}$ and $\exp _{E}$, this yields a split of the given group extension in the category of affine algebraic $F$-groups, so that our theorem is established. 


\section{REFERENCES}

0. A. M. Gleason, Spaces with a compact Lie group of transformations, Proc. Am. Math. Soc., vol. 1 (1950), pp. 35-43.

1. G. Hochschild, The structure of Lie groups, Holden-Day, San Francisco, 1965.

2. C. C. Moore, Group extensions of p-adic and adelic linear groups, I.H.E.S., No. 35 (1968).

3. N. Steenrod, The topology of fibre bundles, Princeton University Press, 1951.

4. W. T. van Est, A generalization of the Cartan-Leray spectral sequence, II, Indag. Math. 20, No. 4 (1958).

Received December 16, 1976.

UNIVERSITY OF CALIFORNIA

BERKELEY, CA 94720

AND

UNIVERSITY OF MICHIGAN

ANN ARBOR, MI 48104 



\section{PACIFIC JOURNAL OF MATHEMATICS}

\section{EDITORS}

RichaRd ARENS (Managing Editor)

University of California

Los Angeles, CA 90024

\section{R. A. Beaumont}

University of Washington

Seattle, WA 98105

C. C. Moore

University of California

Berkeley, CA 94720
J. DugunduI

Department of Mathematics

University of Southern California

Los Angeles, CA 90007

R. Finn AND J. Milgram

Stanford University

Stanford, CA 94305

\section{ASSOCIATE EDITORS}
E. F. BECKENBACH
B. H. NeumanN
F. WOLF
K. YoshidA

\section{SUPPORTING INSTITUTIONS}

UNIVERSITY OF BRITISH COLUMBIA

CALIFORNIA INSTITUTE OF TECHNOLOGY

UNIVERSITY OF CALIFORNIA

MONTANA STATE UNIVERSITY

UNIVERSITY OF NEVADA

NEW MEXICO STATE UNIVERSITY

OREGON STATE UNIVERSITY

UNIVERSITY OF OREGON

OSAKA UNIVERSITY

\author{
UNIVERSITY OF SOUTHERN CALIFORNIA \\ STANFORD UNIVERSITY \\ UNIVERSITY OF HAWAII \\ UNIVERSITY OF TOKYO \\ UNIVERSITY OF UTAH \\ WASHINGTON STATE UNIVERSITY \\ UNIVERSITY OF WASHINGTON \\ AMERICAN MATHEMATICAL SOCIETY
}

The Supporting Institutions listed above contribute to the cost of publication of this Journal, but they are not owners or publishers and have no responsibility for its contents or policies.

Mathematical papers intended for publication in the Pacific Journal of Mathematics should be in typed form or offset-reproduced (not dittoed), double spaced with large margins. Underline Greek letters in red, German in green, and script in blue. The first paragraph or two must be capable of being used separately as a synopsis of the entire paper. Items of the bibliography should not be cited there unless absolutely necessary, in which case they must be identified by author and Journal, rather than by item number. Manuscripts, in duplicate, may be sent to any one of the four editors. Please classify according to the scheme of Math. Reviews, Index to Vol. 39. All other communications should be addressed to the managing editor, or Elaine Barth, University of California, Los Angeles, California, 90024.

100 reprints are provided free for each article, only if page charges have been substantially paid. Additional copies may be obtained at cost in multiples of 50 .

The Pacific Journal of Mathematics is issued monthly as of January 1966. Regular subscription rate: $\$ 72.00$ a year (6 Vols., 12 issues). Special rate: $\$ 36.00$ a year to individual members of supporting institutions.

Subscriptions, orders for back numbers, and changes of address should be sent to Pacific Journal of Mathematics, 103 Highland Boulevard, Berkeley, California, 94708.

PUBLISHED BY PACIFIC JOURNAL OF MATHEMATICS, A NON-PROFIT CORPORATION

Printed at Jerusalem Academic Press, POB 2390, Jerusalem, Israel. 


\section{Pacific Journal of Mathematics}

Vol. 68, No. 2

April, 1977

William Allen Adkins, Aldo Andreotti and John Vincent Leahy, An analogue of Oka's theorem for weakly normal complex spaces ........

Ann K. Boyle, M. G. Deshpande and Edmund H. Feller, On nonsingularly

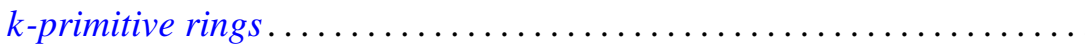

Rolando Basim Chuaqui, Measures invariant under a group of

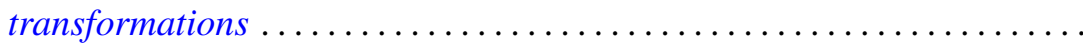

Wendell Dan Curtis and Forrest Miller, Gauge groups and classification of bundles with simple structural group .......................

Garret J. Etgen and Willie Taylor, The essential uniqueness of bounded nonoscillatory solutions of certain even order differential equations

Paul Ezust, On a representation theory for ideal systems

Richard Carl Gilbert, The deficiency index of a third order operator ........

John Norman Ginsburg, $S$-spaces in countably compact spaces using Ostaszewski's method.

Basil Gordon and S. P. Mohanty, On a theorem of Delaunay and some

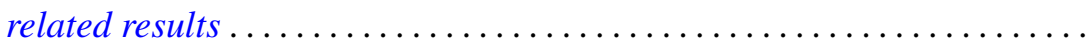

Douglas Lloyd Grant, Topological groups which satisfy an open mapping

theorem.

Charles Lemuel Hagopian, A characterization of solenoids

Kyong Taik Hahn, On completeness of the Bergman metric and its

subordinate metrics. II .

G. Hochschild and David Wheeler Wigner, Abstractly split group extensions.

Gary S. Itzkowitz, Inner invariant subspaces ...............

Jiang Luh and Mohan S. Putcha, A commutativity theorem for

non-associative algebras over a principal ideal domain.

Donald J. Newman and A. R. Reddy, Addendum to: "Rational approximation of $e^{-x}$ on the positive real axis".....

Akio Osada, On the distribution of a-points of a strongly annular function ....

Jeffrey Lynn Spielman, A characterization of the Gaussian distribution in a Hilbert space. .

Robert Moffatt Stephenson Jr., Symmetrizable-closed spaces ...

Peter George Trotter and Takayuki Tamura, Completely semisimple inverse $\Delta$-semigroups admitting principal series . . . . . . . .

Charles Irvin Vinsonhaler and William Jennings Wickless, Torsion free abelian groups quasi-projective over their endomorphism rings...

Frank Arvey Wattenberg, Topologies on the set of closed subsets ... 